\title{
Anionic Fullerenes, Calixarenes, Coronenes and Pyrenes as Activators of Oligo/Polyarginines in Model Membranes and Live Cells
}

\author{
Florent Perret, ${ }^{\dagger}$ Masamichi Nishihara, ${ }^{\dagger}$ Toshihide Takeuchi, ${ }^{\S}$ Shiroh Futaki,${ }^{\S, \#, *}$ Adina \\ N. Lazar, ${ }^{9}$ Anthony W. Coleman, ${ }^{9}$ Naomi Sakai ${ }^{\dagger, *}$ and Stefan Matile ${ }^{\dagger, *}$ \\ ${ }^{\dagger}$ Department of Organic Chemistry, University of Geneva, Geneva, Switzerland, \\ ${ }^{\S}$ Institute for Chemical Research, Kyoto University and ${ }^{\# P R E S T O, ~ J S T, ~ K y o t o, ~ J a p a n, ~}$ \\ and Institut de Biologie et Chimie des Protéines, CNRS UMR 5086, 7 passage du \\ Vercors, F69367, Lyon, France
}

\section{Supporting Information}

General. Reagents for synthesis, 5(6)-carboxyfluorescein (CF), 2-pyrene-1-yl-acetic acid, 6-pyrene-1-yl-caproic acid and lauric acid were purchased from Fluka, poly-Larginine ( $\mathrm{HCl}$ salt, MW 14,000, DP 72), triton X-100, buffers and salts from Sigma, $\mathrm{H}-(\text { Arg) })_{6}-\mathrm{OH}$ (TFA salt) from Bachem, 4-pyrene-1-yl-butyric acid from Acros Organics, coronene from Aldrich, fullerene $\mathrm{C}_{60}$ from Alfa Aesar, egg yolk phosphatidylcholine (EYPC) from Avanti, HATU from Applied Biosystems and mono- $n$-dodecylphosphate from Lancaster. All reactions were performed under $\mathrm{N}_{2}$ or argon atmosphere. Column chromatography was carried out on silica gel 60 (Fluka, 40-63 $\mu \mathrm{m}$ ). Reverse phase column chromatography was performed using ODS (Fluka Silicagel 100 C18-Reverse Phase). Analytical (TLC) and preparative thin layer chromatography (PTLC) were performed in silica gel 60 (Fluka, $0.2 \mathrm{~mm}$ ) and silica gel GF (Analtech, $1000 \mu \mathrm{m}$ ), respectively. Melting points (mp) were recorded on a heating table from Reichert (Austria), IR spectra on a Perkin Elmer Spectrum One FT-IR spectrometer (ATR, Golden Gate) and are reported in $\mathrm{cm}^{-1}$ with band intensities indicated as s (strong), m (medium), w (weak). ESI-MS was performed on a Finnigan MAT SSQ 7000 instrument. ${ }^{1} \mathrm{H}$ and ${ }^{13} \mathrm{C}$ spectra were recorded (as indicated) on either a Bruker $300 \mathrm{MHz}, 400 \mathrm{MHz}$ or $500 \mathrm{MHz}$ spectrometer and are reported as chemical shifts $(\delta)$ in ppm relative to TMS $(\delta=0)$. Spin multiplicities are reported as a singlet (s), doublet (d), triplet (t), with coupling constants $(J)$ given in $\mathrm{Hz}$, or multiplet $(\mathrm{m})$. ${ }^{1} \mathrm{H}$ and ${ }^{13} \mathrm{C}$ resonances were assigned with the aid of additional information from 2D NMR spectra [H,H-COSY, DEPT 135, HSQC and HMBC]. UV-Vis spectra were measured on a Varian Cary 1 Bio spectrophotometer. Fluorescence measurements were preformed on either a FluoroMax-2 or a FluoroMax-3, Jobin Yvon-Spex. The Mini-Extruder with a polycarbonate membrane, pore size $100 \mathrm{~nm}$, used for LUV preparation was from Avanti.

Abbreviations. CF: 5(6)-Carboxyfluorescein; DBU: 1,8-Diazabicyclo[5.4.0] undec-7-ene; DIPEA: $N, N$ '-Diisopropylethylenediamine; DMAP: 4Dimethylaminopyridine; DMF: $N, N$-Dimethylformamide; EYPC-LUVs: Egg yolk phosphatidylcholine large unilamellar vesicles; HATU: $N$-[(dimethylamino)- $1 H$ - 
1,2,3-triazolo[4,5- $b]$ pyridin-1-ylmethylene]- $N$-methylmethanaminiumhexafluorophosphate $N$-oxide; TBDPS: Tetrabutyldiphenylsilyl; THF: Tetrahydrofuran.

Fullerene diacid 1. This compound was prepared from fullerene in overall two steps following previously reported procedures. ${ }^{\mathrm{S} 1}$

4-Coronen-1-yl-butyric acid 2. This compound was prepared from coronene in overall three steps following previously reported procedures. ${ }^{\mathrm{S}}$

4-Pyren-1-yl-butylsulfate 7 (sodium salt). This compound was prepared in overall two steps from 4-pyrene-1-yl-butyric acid $\mathbf{5}$ following previously reported procedures. ${ }^{\mathrm{S} 3}$

Pyrene sulfate 8 (sodium salt). This compound was prepared from 4-pyren-1-ylbutyric acid $\mathbf{5}$ in overall two steps.

4-Pyren-1-yl-butyric acid 12-hydroxydodecyl ester. 1-Pyren-1-yl-butyric acid 5 (1.0 $\mathrm{g}, 3.5 \mathrm{mmol})$ and HATU $(2.4 \mathrm{~g}, 63 \mathrm{mmol})$ in anhydrous THF $(10 \mathrm{ml})$ were added to 1,12-dodecandiol $(7.0 \mathrm{~g}, 35 \mathrm{mmol})$ and $\mathrm{Et}_{3} \mathrm{~N}(3 \mathrm{ml}, 21 \mathrm{mmol})$ in anhydrous THF (20 $\mathrm{ml}$ ) at room temperature. The mixture was stirred at room temperature during 2 hours, diluted with EtOAc $(20 \mathrm{ml})$, washed with water $(2 \times 50 \mathrm{ml})$, dried with $\mathrm{MgSO}_{4}$ and concentrated in vacuo. Purification by column chromatography $\left(\mathrm{CH}_{2} \mathrm{Cl}_{2}: \mathrm{MeOH}\right.$ 20:1, $\left.R_{\mathrm{f}}=0.6\right)$ gave pure 4-pyren-1-yl-butyric acid 12-hydroxydodecyl ester $(900 \mathrm{mg}$, $55 \%)$ as a colorless solid. mp: $>230{ }^{\circ} \mathrm{C} ;{ }^{1} \mathrm{H}$ NMR $\left(300 \mathrm{MHz}, \mathrm{CD}_{3} \mathrm{OD}\right): \delta 8.35\left(\mathrm{~d},{ }^{3} \mathrm{~J}\right.$ $(\mathrm{H}, \mathrm{H})=9.2 \mathrm{~Hz}, 1 \mathrm{H}), 8.20-8.12(\mathrm{~m}, 4 \mathrm{H}), 8.04-7.98(\mathrm{~m}, 3 \mathrm{H}), 7.89\left(\mathrm{~d},{ }^{3} J(\mathrm{H}, \mathrm{H})=7.7\right.$ $\mathrm{Hz}, 1 \mathrm{H}), 4.03\left(\mathrm{t},{ }^{3} J(\mathrm{H}, \mathrm{H})=6.6 \mathrm{~Hz}, 2 \mathrm{H}\right), 3.51\left(\mathrm{t},{ }^{3} J(\mathrm{H}, \mathrm{H})=6.6 \mathrm{~Hz}, 2 \mathrm{H}\right), 3.40\left(\mathrm{t},{ }^{3} J\right.$ $(\mathrm{H}, \mathrm{H})=7.7 \mathrm{~Hz}, 2 \mathrm{H}), 2.49\left(\mathrm{t},{ }^{3} J(\mathrm{H}, \mathrm{H})=7.0 \mathrm{~Hz}, 2 \mathrm{H}\right), 2.17\left(\mathrm{tt},{ }^{3} J(\mathrm{H}, \mathrm{H})=7.0 \mathrm{~Hz},{ }^{3} J\right.$ $(\mathrm{H}, \mathrm{H})=7.7 \mathrm{~Hz}, 2 \mathrm{H}), 1.57\left(\mathrm{tt},{ }^{3} J(\mathrm{H}, \mathrm{H})=7.0 \mathrm{~Hz},{ }^{3} J(\mathrm{H}, \mathrm{H})=6.6 \mathrm{~Hz}, 2 \mathrm{H}\right), 1.48\left(\mathrm{tt},{ }^{3} J\right.$ $\left.(\mathrm{H}, \mathrm{H})=6.6 \mathrm{~Hz},{ }^{3} \mathrm{~J}(\mathrm{H}, \mathrm{H})=6.8 \mathrm{~Hz}, 2 \mathrm{H}\right), 1.27(\mathrm{~m}, 16 \mathrm{H}) ;{ }^{13} \mathrm{C} \mathrm{NMR}\left(75 \mathrm{MHz}, \mathrm{CD}_{3} \mathrm{OD}\right)$ : ठ 172.2 (s), 136.7 (s), 131.0 (s), 130.6 (s), 129.8 (s), 128.2 (s), 127.2 (d), 127.1 (d), 127.0 (d), 127.9 (d), 126.1 (d), 125.5 (d), 124.5 (d), 124.4 (s), 124.4 (s), 124.3 (d), $123.2(\mathrm{~d}), 66.7(\mathrm{t}), 63.1(\mathrm{t}), 33.4(\mathrm{t}), 33.3(\mathrm{t}), 33.2(\mathrm{t}), 30.4(\mathrm{t}), 30.4(\mathrm{t}), 30.3(\mathrm{t}), 30.2$ $(\mathrm{t}), 30.2(\mathrm{t}), 30.0(\mathrm{t}), 29.9(\mathrm{t}), 27.8(\mathrm{t}), 26.6(\mathrm{t}), 26.1(\mathrm{t}) ; \mathrm{MS}(\mathrm{ACPI}, \mathrm{MeOH}): \mathrm{m} / \mathrm{z}(\%)$ $473(100)[\mathrm{M}]^{+}$.

Pyrene sulfate 8 (sodium salt). A solution of 4-pyren-1-yl-butyric acid 12hydroxydodecyl ester $(200 \mathrm{mg}, 0.42 \mathrm{mmol})$ in anhydrous ether $(0.4 \mathrm{ml})$ was transferred slowly to a solution of chlorosulfuric acid $(29 \mu 1,0.42 \mathrm{mmol})$ in anhydrous ether $(2 \mathrm{ml})$ at $0{ }^{\circ} \mathrm{C}$. The mixture was stirred for 1 hour at room temperature and purged with nitrogen to remove $\mathrm{HCl}$. The mixture was diluted with water $(4 \mathrm{ml}), 1 \mathrm{M}$ $\mathrm{NaOH}$ (to reach $\mathrm{pH} \sim 7)$ and 2-propanol $(5 \mathrm{ml})$, washed with hexane $(2 \times 10 \mathrm{ml})$ and concentrated in vacuo. Purification by reverse phase column chromatography ( $\left.\mathrm{MeOH}, R_{\mathrm{f}}=0.8\right)$ yielded pure $8(220 \mathrm{mg}, 90 \%)$ as a yellowish solid. $\mathrm{mp}:>230{ }^{\circ} \mathrm{C}$; ${ }^{1} \mathrm{H}$ NMR $\left(300 \mathrm{MHz}, \mathrm{CD}_{3} \mathrm{OD}\right): \delta 8.27\left(\mathrm{~d},{ }^{3} J(\mathrm{H}, \mathrm{H})=9.2 \mathrm{~Hz}, 1 \mathrm{H}\right), 8.11-8.03(\mathrm{~m}, 4 \mathrm{H})$, 7.96-7.90 (m, 3H), $7.80\left(\mathrm{~d},{ }^{3} J(\mathrm{H}, \mathrm{H})=7.7 \mathrm{~Hz}, 1 \mathrm{H}\right), 3.94\left(\mathrm{t},{ }^{3} J(\mathrm{H}, \mathrm{H})=6.6 \mathrm{~Hz}, 2 \mathrm{H}\right)$, $3.86\left(\mathrm{t},{ }^{3} J(\mathrm{H}, \mathrm{H})=6.6 \mathrm{~Hz}, 2 \mathrm{H}\right), 3.30\left(\mathrm{t},{ }^{3} J(\mathrm{H}, \mathrm{H})=7.7 \mathrm{~Hz}, 2 \mathrm{H}\right), 2.40\left(\mathrm{t},{ }^{3} J(\mathrm{H}, \mathrm{H})=7.2\right.$ $\mathrm{Hz}, 2 \mathrm{H}), 2.08\left(\mathrm{tt},{ }^{3} J(\mathrm{H}, \mathrm{H})=7.5 \mathrm{~Hz}, 2 \mathrm{H}\right), 1.50\left(\mathrm{tt},{ }^{3} J(\mathrm{H}, \mathrm{H})=7.0 \mathrm{~Hz}, 2 \mathrm{H}\right), 1.48\left(\mathrm{tt},{ }^{3} J\right.$ 
$(\mathrm{H}, \mathrm{H})=7.0 \mathrm{~Hz}, 2 \mathrm{H}), 1.18(\mathrm{~m}, 16 \mathrm{H}) ;{ }^{13} \mathrm{C}$ NMR $\left(75 \mathrm{MHz}, \mathrm{CD}_{3} \mathrm{OD}\right): \delta 172.2(\mathrm{~s}), 136.7$ (s), 131.0 (s), 130.6 (s), 129.8 (s), 128.2 (s), 127.2 (d), 127.1 (d), 127.0 (d), 127.9 (d), 126.1 (d), 125.5 (d), 124.5 (d), 124.4 (s), 124.4 (s), 124.3 (d), 123.2 (d), 70.1 (d), 66.7 $(\mathrm{t}), 33.4(\mathrm{t}), 33.3(\mathrm{t}), 30.4(\mathrm{t}), 30.4(\mathrm{t}), 30.3(\mathrm{t}), 30.2(\mathrm{t}), 30.2(\mathrm{t}), 30.0(\mathrm{t}), 29.9(\mathrm{t}), 29.8$ $(\mathrm{t}), 27.8(\mathrm{t}), 26.6(\mathrm{t}), 26.1(\mathrm{t})$; MS (ESI negative mode, MeOH): $\mathrm{m} / \mathrm{z}(\%) 551(100)[\mathrm{M}$ $-\mathrm{Na}]^{-}$.

Calix[4]arene carboxylate 3. This compound was prepared following previously reported procedures. ${ }^{\mathrm{S}}$

Calix[4]arene diphosphate 11. This compound was prepared following previously reported procedures. ${ }^{\text {S4 }}$

Stock solutions. The following stock solutions were prepared and their $\mathrm{pH}$ was adjusted $^{\mathrm{S5}}$ if necessary: 1 in DMSO / water 1:1, 2 in DMSO / water 9:1, other anions in water.

EYPC-LUVs $\supset$ CF. Stock solutions of large unilamellar vesicles composed of egg yolk phosphatidylcholine loaded with $\mathrm{CF}$ were prepared by freeze-thaw-extrusion following the previously described method $^{\mathrm{S} 6}$ without change. Final conditions: $\sim 1.3$ mM EYPC; inside: $10 \mathrm{mM}$ sodium phosphate, $50 \mathrm{mM} \mathrm{CF}, 10 \mathrm{mM} \mathrm{NaCl}, \mathrm{pH} 7.4$; outside: $10 \mathrm{mM}$ sodium phosphate, $107 \mathrm{mM} \mathrm{NaCl}, \mathrm{pH} 7.4$.

CF efflux assay, general procedure (adapted from S6). EYPC-LUVs $\supset C F(20 \mu 1)$ were added to gently stirred, thermostated buffer $(1.94 \mathrm{ml}, 10 \mathrm{mM}$ sodium phosphate, $107 \mathrm{mM} \mathrm{NaCl}, \mathrm{pH}$ 7.4) in a fluorescence cuvette. The time course of CF efflux was followed at $\lambda_{\mathrm{em}} 517 \mathrm{~nm}\left(\lambda_{\mathrm{ex}} 490 \mathrm{~nm}\right)$ as a function of time during the addition of anion (20 $\mu \mathrm{l}$ of different concentrated solutions), polyarginine (20 $\mu \mathrm{l}$ of $25 \mu \mathrm{M}$ in $\mathrm{H}_{2} \mathrm{O}$, final concentration $250 \mathrm{nM}$ ) and triton X-100 (0.024\% final) at the end of each experiment. Fluorescence time courses were normalized to fractional emission intensity $I$ using equation [S1]

$$
I=\left(F_{\mathrm{t}}-F_{0}\right) /\left(F_{\infty}-F_{0}\right)
$$

where $F_{0}=F_{\mathrm{t}}$ at polyarginine addition, $F_{\infty}=F_{\mathrm{t}}$ at saturation after lysis. Effective concentrations $E C_{50}$ and Hill coefficients were determined from $I$ just before lysis as a function of anion concentration and fitted to the Hill equation [S2]

$$
I=I_{0}+\left(I_{\infty}-I_{0}\right) /\left\{1+\left(\mathrm{C}_{\text {anion }} / E C_{50}\right)^{n}\right\}
$$

where $I_{0}$ is $I$ without anion, $I_{\infty}$ is value at saturation, $\mathrm{C}_{\text {anion }}$ is the anion concentration in cuvette and $n$ is the Hill coefficient, to give the effective concentration $E C_{50}$. 
Table S2. Summary of anion-activation of polyarginine $(250 \mathrm{nM})$

\begin{tabular}{crllllllll}
\hline Activator & \multicolumn{2}{c}{$E C_{50}(\mu \mathrm{M})$} & \multicolumn{3}{c}{$n$} & \multicolumn{3}{c}{$Y_{\max }(\%)$} \\
\hline $\mathbf{1}$ & 0.05 & $\mathbf{\pm}$ & 0.002 & 2.6 & \pm & 0.3 & 48 & \pm & 1 \\
$\mathbf{2}$ & 4.3 & \pm & 0.3 & 1.4 & \pm & 0.1 & 65 & \pm & 2 \\
$\mathbf{3}$ & 3.1 & \pm & 0.2 & 2.5 & \pm & 0.3 & 68 & \pm & 2 \\
$\mathbf{4}$ & 86 & \pm & 3 & 4.3 & \pm & 0.5 & 80 & \pm & 3 \\
$\mathbf{5}$ & 44 & \pm & 2 & 2.2 & \pm & 0.2 & 78 & \pm & 2 \\
$\mathbf{6}$ & 9.3 & \pm & 0.7 & 2.9 & \pm & 0.7 & 55 & \pm & 4 \\
$\mathbf{7}$ & 6.7 & \pm & 0.6 & 2.2 & \pm & 0.5 & 48 & \pm & 3 \\
$\mathbf{8}$ & 2.2 & \pm & 0.2 & 1.9 & \pm & 0.3 & 46 & \pm & 2 \\
$\mathbf{9}$ & 19 & \pm & 1 & 4.2 & \pm & 1 & 61 & \pm & 3 \\
$\mathbf{1 0}$ & 34 & \pm & 3.8 & \pm & 0.4 & 10 & \pm & 1 \\
\hline
\end{tabular}

Alexa 488-labeled $\mathbf{R}_{\mathbf{8}} \cdot \mathrm{H}-(\mathrm{Arg})_{8}$-Gly-Cys- $\mathrm{NH}_{2}{ }^{\mathrm{S} 7}$ was treated with Alexa $488 \mathrm{C}_{5}$ maleimide sodium salt (Molecular Probes) (1.5 eq) in DMF-MeOH (1:1) for $1.5 \mathrm{~h}$, and the product was purified by RP-HPLC. MALDI-TOFMS 2127.2 [Calcd. for $[\mathrm{M}+\mathrm{H}]^{+}:$2126.4].

Cell culture. Human cervical cancer-derived HeLa cells were cultured as exponentially growing subconfluent monolayers on $60-\mathrm{mm}$ dishes in alpha-minimum essential medium ( $\alpha$-MEM) supplemented with $10 \%(\mathrm{v} / \mathrm{v})$ calf serum. A subculture was performed every 3-4 days.

Flow cytometry. $1.5 \times 10^{5} \mathrm{HeLa}$ cells in $1.5 \mathrm{ml}$ of $\alpha$-MEM containing $10 \%(\mathrm{v} / \mathrm{v})$ calf serum were plated into 12-well microplates (Iwaki) and cultured for $48 \mathrm{~h}$. After removing the medium, the cells were washed twice with PBS. The cells were then incubated for $5 \mathrm{~min}$ at $37^{\circ} \mathrm{C}$ in $300 \mu \mathrm{l}$ of PBS in the presence or absence of a pyrenebutyrate 5 prior to addition of $100 \mu$ l of PBS containing the R8 peptide (final peptide concentration, $5 \mu \mathrm{M}$ ). After a 15 -min incubation with the peptide at $37^{\circ} \mathrm{C}$, the cells were washed three times with PBS, and then incubated with $0.01 \%$ trypsin $(400$ $\mu 1)$ for $10 \mathrm{~min}$ at $37^{\circ} \mathrm{C}$. After addition of $600 \mu \mathrm{l}$ of PBS, the cells were centrifuged at 2,000 rpm for $5 \mathrm{~min}$. The cell pellet was suspended and washed twice with $1 \mathrm{ml}$ of PBS, and finally resuspended in $1 \mathrm{ml}$ of PBS to perform fluorescence analysis with a FACScalibur (BD Biosciences) flow cytometer using a 488-nm laser excitation and a 515-545 nm emission filter. Each sample was analyzed 10,000 events.

(S1) (a) Bingel, C. Chem. Ber. 1993, 126, 1957-1959. (b) Lamparth, I.; Hirsch, A. J. Chem. Soc. Chem. Commun. 1994, 14, 1727-1728.

(S2) Davenport, L.; Shen, B.; Joseph, T. W.; Straher, M. P. Chem. Phys. Lipids 2001, 109, 145-156.

(S3) Gorteau, V.; Perret, F.; Bollot, G.; Mareda, J.; Lazar, A. N.; Coleman, A. W.; Tran, D.-H.; Sakai, N.; Matile, S. J. Am. Chem. Soc. 2004, 126, 13592-13593.

(S4) (a) Da Silva, E.; Lazar, A. N.; Coleman, A. W. J. Drug Del. Sci. Tech. 2004, 14, 3-20. (b) Iwamoto, K.; Shinkai, S. J. Org. Chem. 1992, 57, 7066-7073. (c) 
McKervey, M. A.; Seward, E. M.; Ferguson, G.; Ruhl, B.; Harris, S. J. J. Chem. Soc. Chem. Commun. 1985, 7, 388-390.

(S5) Das, G.; Onouchi, H.; Yashima, E.; Sakai, N.; Matile, S. ChemBioChem 2002, 3, 1089-1096.

(S6) Sakai, N.; Matile, S. J. Am. Chem. Soc. 2003, 125, 14348-14356.

(S7) Futaki, S.; Suzuki, T.; Ohashi, W.; Yagami, T.; Tanaka, S.; Ueda, K.; Sugiura, Y. J. Biol. Chem. 2001, 276, 5836-5840. 